\title{
CHAOTIC NUMERICS FROM AN INTEGRABLE HAMILTONIAN SYSTEM
}

\author{
KEVIN HOCKETT
}

(Communicated by Kenneth R. Meyer)

\begin{abstract}
We study the dynamics of the map $E$ obtained by applying Euler's method with stepsize $h$ to the central force problem. We prove that, for any $h>0$, the nonwandering set of $E$ contains a subset on which the dynamics of $E$ are topologically semiconjugate to a subshift of finite type. The subshift has positive topological entropy, hence so does $E$.
\end{abstract}

\section{INTRODUCTION}

Hamiltonian dynamical systems, like any dynamical system with special properties, present certain difficulties with respect to their numerical study. Recently there has been a great deal of effort devoted to the development of numerical algorithms which preserve the Hamiltonian structure of these systems, i.e., symplectic integrators (see, e.g., Channell and Scovel [1988], Neri [1988], Ruth [1983]). These algorithms offer many advantages over nonsymplectic schemes: they better preserve the total energy, can be designed to preserve symmetries of the system, etc.

At the same time there has been substantial interest in understanding the global dynamical behavior of some of the classical algorithms of analysis. Studies include the dynamics of Newton's method (e.g., Curry, et al. [1983], Smale [1985], Saari and Urenko [1984]), the geometry and dynamics of the classical QR algorithm (Shub and Vasquez [1984]), 'computational chaos' occurring when the stepsize of a numerical method is taken too large (Lorenz [1988]), and shadowing theorems for numerical orbits of non-uniformly hyperbolic maps (Hammel, et al. [1988]).

In this paper we provide a simple example which serves to illustrate the extent to which spurious numerical results may be obtained by failing to take into account special properties of a system. Specifically, we investigate the global dynamics of the map $E$ obtained by applying Euler's method with stepsize $h$ to the equations of motion of the central force problem. This system is Hamiltonian and the vector field possesses singularities. We show that for all

Received by the editors March 6, 1989.

1980 Mathematics Subject Classification (1985 Revision). Primary 58F08, 58F14.

(C) 1990 American Mathematical Society $0002-9939 / 90 \$ 1.00+\$ .25$ per page 
$h>0$, the map $E$ admits 'chaotic' dynamics (indeed, has positive topological entropy). This is in sharp contrast to the dynamics of the underlying system which is well known to be integrable.

Euler's method is clearly not the algorithm of choice for any serious numerical study, and there is certainly no need to numerically integrate an integrable system. Nonetheless, it is striking that the dynamics of $E$ differ so radically from those of the central force problem and, moreover, that this anomolous behavior cannot be eliminated by choosing the stepsize small.

\section{THE CENTRAL FORCE PROBLEM AND THE EULER MAP}

The planar motion of a particle which is attracted to the origin by a Newtonian central force is described by the equation

$$
\ddot{\vec{r}}=-\mu r^{-3} \vec{r} \text {. }
$$

Here $\vec{r}$ is the position vector of the particle and $\mu$ is a positive constant depending on the units chosen and the mass of the particle. Expressed in polar coordinates this system of equations is

$$
\begin{gathered}
\ddot{r}-r \dot{\theta}^{2}=-\mu r^{-2}, \\
r \ddot{\theta}+2 \dot{r} \dot{\theta}=0 .
\end{gathered}
$$

From the second of these equations one finds that

$$
p_{\theta}=r^{2} \dot{\theta}
$$

is a conserved quantity for the system. This is essentially the statement that angular momentum is conserved.

Using (2.3), equations (2.2) are seen to be equivalent to the first order system

$$
\begin{gathered}
\dot{r}=p_{r}=\partial H / \partial p_{r}, \\
\dot{\theta}=p_{\theta} r^{-2}=\partial H / \partial p_{\theta}, \\
\dot{p}_{r}=p_{\theta}^{2} r^{-3}-\mu r^{-2}=-\partial H / \partial r, \\
\dot{p}_{\theta}=0=-\partial H / \partial \theta .
\end{gathered}
$$

Here $H$ is the Hamiltonian (or total energy) of the system,

$$
H\left(r, \theta, p_{r}, p_{\theta}\right)=\frac{1}{2}\left(p_{r}^{2}+p_{\theta}^{2} r^{-2}\right)-\mu r^{-1},
$$

and the equations (2.4a-d) are clearly in Hamiltonian form (see, e.g., Arnold [1978]). That is, the equations (2.4a-d) have the form $\dot{x}=J \nabla H(x), x \in R^{2 n}$ where

$$
J=\left(\begin{array}{cc}
0 & I \\
-I & 0
\end{array}\right)
$$

and $I$ is the $n \times n$ identity matrix. 
If we apply Euler's method with stepsize $h$ to (2.4) we obtain the Euler map $\mathscr{E}_{h}:$

$$
\left[\begin{array}{c}
r \\
\theta \\
p_{r} \\
p_{\theta}
\end{array}\right] \stackrel{\mathscr{E}_{h}}{\longrightarrow}\left[\begin{array}{c}
r+h p_{r} \\
\theta+h p_{\theta} r^{-2} \\
p_{r}+h r^{-3}\left(p_{\theta}^{2}-\mu r\right) \\
p_{\theta}
\end{array}\right]
$$

It is this map that one iterates when using Euler's method to obtain approximate solutions of the system (2.4). We make two observations about $\mathscr{E}_{h}$. First, the map preserves the fact that $p_{\theta}$ is a conserved quantity for the system (2.4). We are therefore free to set $p_{\theta}=$ const. and reduce $\mathscr{E}_{h}$ to a mapping in 3dimensions. Second, we observe that the evolution of the $r$-and $p_{r}$-coordinates under iteration of $\mathscr{E}_{h}$ do not depend on the evolution of the $\theta$-coordinate. We will therefore ignore the behavior of the $\theta$-coordinate, and devote the remainder of this paper to studying the dynamics of the reduced Euler map E:

$$
\left[\begin{array}{l}
r \\
p
\end{array}\right] \stackrel{E}{\longrightarrow}\left[\begin{array}{c}
r+A p \\
p+r^{-3}(B-C r)
\end{array}\right] .
$$

For convenience we have introduced the notation $A=h, B=h p_{\theta}^{2}, C=h \mu$ and have dropped the subscript $r$ from $p_{r}$. Observe that the corresponding system of differential equations

$$
\begin{gathered}
\dot{r}=p=\partial H_{1} / \partial p \\
\dot{p}=p_{\theta}^{2} r^{-3}-\mu r^{-2}=-\partial H_{1} / \partial r
\end{gathered}
$$

is a Hamiltonian system with Hamiltonian function $H_{1}(r, p)=\frac{1}{2}\left(p^{2}+p_{\theta}^{2} r^{-2}\right)-$ $\mu r^{-1}$, where $p_{\theta}$ is a fixed constant. Equations (2.8) describe the radial component of the motion of the particle.

\section{The Dynamics of E}

Before stating our main result we recall some basic definitions. See, e.g., Bowen [1978] or Shub [1987] for general background. Let $f: X \rightarrow X$ be a continuous mapping of a topological space $X$. A point $x \in X$ is wandering if there exists a neighborhood $U$ of $x$ such that $f^{k}(U) \cap U=\varnothing$ for all $k \geq 1$. A point which does not wander is called nonwandering, and the nonwandering set $\Omega_{f}$ (the set of all nonwandering points) is closed and satifies $f\left(\Omega_{f}\right) \subset \Omega_{f}$.

Let $S_{n}=\{1,2, \ldots, n\}$ with the discrete topology and let $\Sigma_{n}=\prod_{0}^{\infty} S_{n}$ with the product topology. $\Sigma_{n}$ is the space of semi-infinite sequences of the symbols $S_{n}$. The space $\Sigma_{n}$ is metrizable, and the metric $d(a, b)=\Sigma_{k=0}^{\infty}\left|a_{k}-b_{k}\right| / 2^{k+1}$ induces the product topology on $\Sigma_{n}$. We define the shift map $\sigma: \Sigma_{n} \rightarrow \Sigma_{n}$ by $\sigma\left(a_{0} a_{1} a_{2} \cdots\right)=a_{1} a_{2} \cdots$. The shift is easily seen to be a continuous n-to1 map of $\Sigma_{n}$. Now let $\mathscr{A}$ be a square matrix of $0^{\prime} s$ and $1^{\prime} s$ and define $\Sigma_{\mathscr{Q}}=\left\{a=a_{0} a_{1} a_{2} \cdots \in \Sigma_{n} \mid \mathscr{A}_{a_{i}, a_{i+1}}=1, i=1,2, \ldots\right\}$. Letting $\sigma_{\mathscr{A}}$ denote the restriction of $\sigma$ to $\Sigma_{\mathscr{A}}$, we call the pair $\left(\Sigma_{\mathscr{A}}, \sigma_{\mathscr{A}}\right)$ a subshift of finite type. The 
matrix $\mathscr{A}$ is called the transition matrix of the subshift. $\mathscr{A}$ can be shown to have at least one positive eigenvalue $\lambda$ (Gantmacher [1960]).

Finally we recall the definition of topological entropy. We shall assume that $X$ is a compact metric space. A set $S \subset X$ is $(n, \epsilon)$-separated for $f$ if $x, y \in S$ implies $d\left(f^{k}(x), f^{k}(y)\right)>\epsilon$ for some $0 \leq k<n$. Let $s(n, \epsilon)=$ $\max \{\operatorname{card} S \mid S$ is $(n, \epsilon)$-separated for $f\}$ and define the growth rate $h(f, \epsilon)=$ $\lim \sup _{n \rightarrow \infty} \ln s(n, \epsilon) / n$. The topological entropy of $f$ is $h(f)=\lim _{\epsilon \rightarrow 0} h(f, \epsilon)$. In general, $h(f)$ is difficult to compute. But for a subshift of finite type we have $h\left(\sigma_{\mathscr{A}}\right)=\ln \left(\lambda^{*}\right)$, where $\lambda^{*}$ is the largest eigenvalue of the transition matrix $\mathscr{A}$ (Bowen [1978]).

Theorem A. Let $E$ be as in (2.7). Then for all $A>0$ the nonwandering set $\Omega$ of $E$ contains a subset $\Lambda$ such that the action of $E$ on $\Lambda$ is topologically semiconjugate to the subshift of finite type with transition matrix

$$
\mathscr{A}=\left[\begin{array}{lll}
0 & 1 & 0 \\
0 & 0 & 1 \\
1 & 1 & 0
\end{array}\right] .
$$

That is, there exists a continuous surjective map $k$ such that the following diagram commutes.

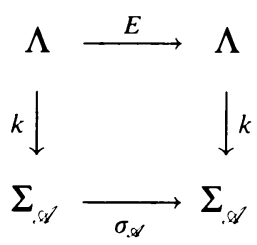

Moreover, $\left(\Sigma_{\mathscr{G}}, \sigma_{, \mathscr{G}}\right)$ has topological entropy $h\left(\sigma_{\mathscr{G}}\right)>0$, hence $h(E)>0$.

To prove the theorem we construct three compact sets $\mathscr{K}_{1}, \mathscr{K}_{2}, \mathscr{K}_{3}$ in $R^{2}-$ $\{r=0\}$ such that

$$
E\left(\mathscr{K}_{1}\right) \supset \mathscr{K}_{2}, \quad E\left(\mathscr{K}_{2}\right) \supset \mathscr{K}_{3}, \quad E\left(\mathscr{K}_{3}\right) \supset \mathscr{K}_{1} \cup \mathscr{K}_{2} .
$$

Define $\Lambda_{n}=\left(\mathscr{K}_{1} \cup \mathscr{K}_{2} \cup \mathscr{K}_{3}\right) \cap E^{-n}\left(\mathscr{K}_{1} \cup \mathscr{K}_{2} \cup \mathscr{K}_{3}\right)$. Then by (3.0) each $\Lambda_{n}$ is a nonempty compact subset of $\mathscr{K}_{1} \cup \mathscr{K}_{2} \cup \mathscr{K}_{3}$. Moreover, $\Lambda_{n} \supset \Lambda_{n+1}$ for all $n$, and thus $\Lambda=\bigcap_{n=0}^{\infty} \Lambda_{n}$ is a nonempty compact $E$-invariant subset of the plane. We associate with $\Lambda$ the $3 \times 3$ matrix $\mathscr{A}$ defined by

$$
\mathscr{A}_{i j}= \begin{cases}1 & \text { if } E\left(\mathscr{K}_{i}\right) \supset \mathscr{K}_{j}, \\ 0 & \text { otherwise. }\end{cases}
$$

By (3.0), $\mathscr{A}$ is the matrix given in the statement of the theorem.

For each $x \in \Lambda$ we define $k(x) \in \Sigma_{\mathscr{A}}$ by $(k(x))_{i}=j$ if $E^{i}(x) \in \mathscr{K}_{j}, i \geq 0$, $j=1,2,3$. The map $k: \Lambda \rightarrow \Sigma_{\mathscr{A}}$ is surjective by the definition of $\mathscr{A}$ and is easily seen to be continuous and to commute with the shift $\sigma_{\mathscr{O Q}}$.

Finally we observe that $\mathscr{A}$ has characteristic polynomial $p(\lambda)=1-\lambda\left(\lambda^{2}-1\right)$ which can be seen to have a root $\lambda_{*}$ satisfying $1<\lambda_{*}<2$. Since the 
topological entropy of $\left(\Sigma_{\mathscr{A}}, \sigma_{\mathscr{A}}\right)$ is $h\left(\sigma_{\mathscr{A}}\right)=\ln \left(\lambda^{*}\right)$, where $\lambda^{*}$ is the largest positive eigenvalue of $\mathscr{A}$, we find $h\left(\sigma_{\mathscr{A}}\right) \geq \ln \left(\lambda_{*}\right)>0$.

We begin our construction of the sets $\mathscr{K}_{1}, \mathscr{K}_{2}, \mathscr{K}_{3}$ by defining two regions in the plane, the 'triangle'

$$
T:\{(r, p) \mid r>0, p \leq-r / A\},
$$

and the ' $\beta$-chisel'

$$
C_{\beta}:\{(r, p) \mid-\beta \leq r<0, \beta>0\} \cap\{(r, p) \mid r<0, p \geq-r / A\}
$$

(see Figure 1).

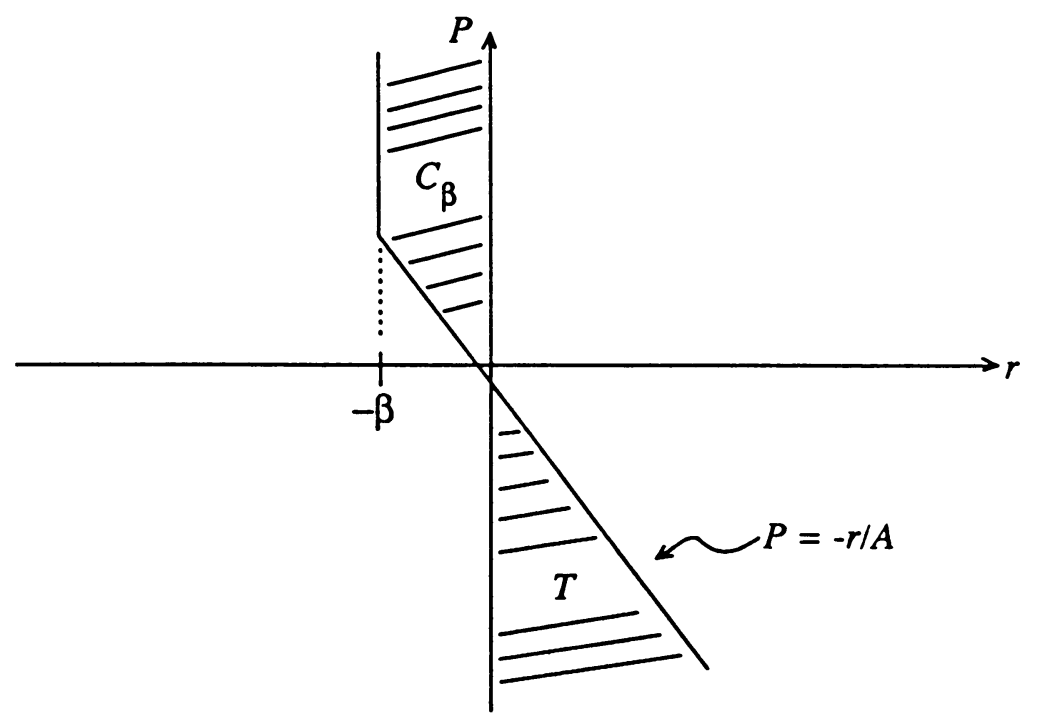

Figure 1. The Regions $T$ AND $C_{\beta}$.

Proposition 1. The triangle $T$ has a pre-image $T^{-1}$ in the right half-plane $r>0$. Moreover, there exists $K>0$ such that if $(r, p) \in T^{-1}$, then $r>0$ and $p<K$. Proof. We prove this proposition in two parts.

Lemma 1. The ray $\mathscr{L}_{k}=\{r=k, p \leq-k / A\}, k>0$ has a pre-image in the right half-plane.

Proof of Lemma 1. We must show that the system of equations

$$
\begin{gathered}
r+A p=k, \\
p+r^{-3}(B-C r)=p_{1}
\end{gathered}
$$

always has a solution $(r, p)$ with $r>0$. 
From (3.1a) we find $p=(k-r) / A$. Now the image of $(r,(k-r) / A)$ under $E$ is

$$
E(r,(k-r) / A)=\left(k,(k-r) / A+r^{-3}(B-C r)\right) .
$$

Define

$$
f_{1}(r)=(k-r) / A+r^{-3}(B-C r) .
$$

Then $f_{1}(r)$ is continuous for $r>0, \lim _{r \rightarrow 0^{+}} f_{1}(r)=+\infty$ and $\lim _{r \rightarrow+\infty} f_{1}(r)=$ $-\infty$. Since $f_{1}$ is onto $(-\infty,+\infty)$, the ray $p=(k-r) / A, r>0$ maps onto $\mathscr{L}_{k}$ under $E$, hence it contains a pre-image of $\mathscr{L}_{k}$.

Letting $k$ vary over all positive numbers, the $\mathscr{L}_{k}$ cover $T$, and their preimages $\mathscr{L}_{k}^{-1}$ cover a pre-image $T^{-1}$ of $T$, establishing the first statement of Proposition 1.

The second statement follows from

Lemma 2. There exists $K>0$ such that if $p \geq K$ then $E(r, p)$ cannot lie in the fourth quadrant $\{r>0, p<0\}$.

Proof of Lemma 2. Consider the image of the horizontal line $p=K, K>0$ :

$$
(r, K) \stackrel{E}{\longrightarrow}\left(r+A K, K+r^{-3}(B-C r)\right)=\left(r_{1}, p_{1}\right) .
$$

Let $f_{2}(r)=r^{-3}(C r-B)$. One easily establishes that $r_{*}=3 B / 2 C$ is a global maximum for $f_{2}$ and that $f_{2}\left(r_{*}\right)=4 C^{3} / 27 B^{2}$. Thus for $K>4 C^{3} / 27 B^{2}$ we have $p_{1}=K+r^{-3}(B-C r)>0$, and $E(r, K)$ is not in the fourth quadrant.

This completes the proof of Proposition 1.

Next we observe that the $\beta$-chisel $C_{\beta}$ is mapped by $E$ onto the region shown in Figure 2. To see this, note that the vertical ray $r=-\beta, p \geq-\beta / A, \beta>0$ maps onto the ray

$$
\begin{gathered}
p=r / A+p_{\beta}, \quad r>0, \\
p_{\beta}=\beta / A-\beta^{-3}(B+C \beta) .
\end{gathered}
$$

Since $p_{\beta} \rightarrow+\infty$ as $\beta \rightarrow+\infty$ and $p_{\beta} \rightarrow-\infty$ as $\beta \rightarrow 0^{+}$, by Lemma 2 we see that for $\beta>0$ sufficiently large, $E\left(C_{\beta}\right) \supset T \cup T^{-1}$. Fix such a $\beta$, say $\beta=\beta_{1}$.

Consider the image of the line segment $\mathscr{L}_{\alpha}:\{(r,-\alpha) \mid \alpha>0,0<r \leq \alpha A\}$ under $E$ which is given by the graph of the function

$$
f_{3}(r)=-\alpha+(r+A \alpha)^{-3}[B-C(r+A \alpha)], \quad-A \alpha<r \leq 0
$$

(see Figure 3).

We note several features of $E\left(\mathscr{L}_{\alpha}\right)$. First, $f_{3}(0)=-\alpha+(A \alpha)^{-3}[B-C A \alpha]<0$ if $\alpha>0$ is sufficiently large, say $\alpha=\alpha_{3}$. Next, note that $f_{3}(r) \rightarrow+\infty$ as $r \rightarrow(-A \alpha)^{+}$. Moreover, if $\beta_{1}$ is chosen as above then $-A \alpha<-\beta_{1}$ for $\alpha>0$ 


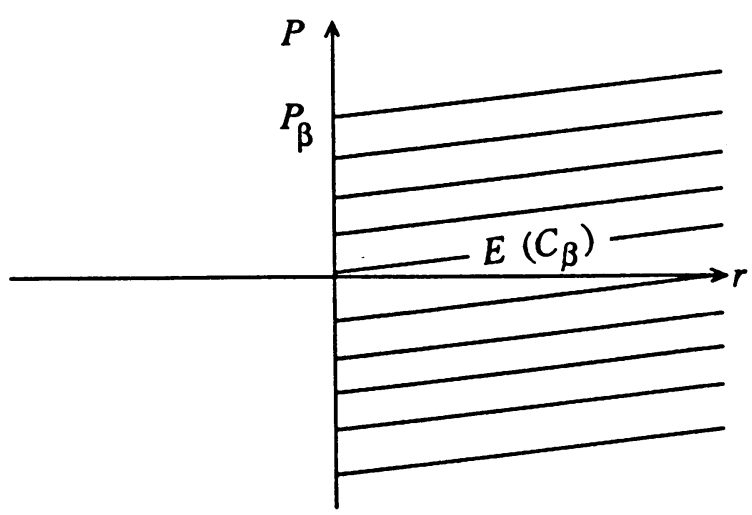

Figure 2. The image $E\left(C_{\beta}\right)$ Of The $\beta$-Chisel.

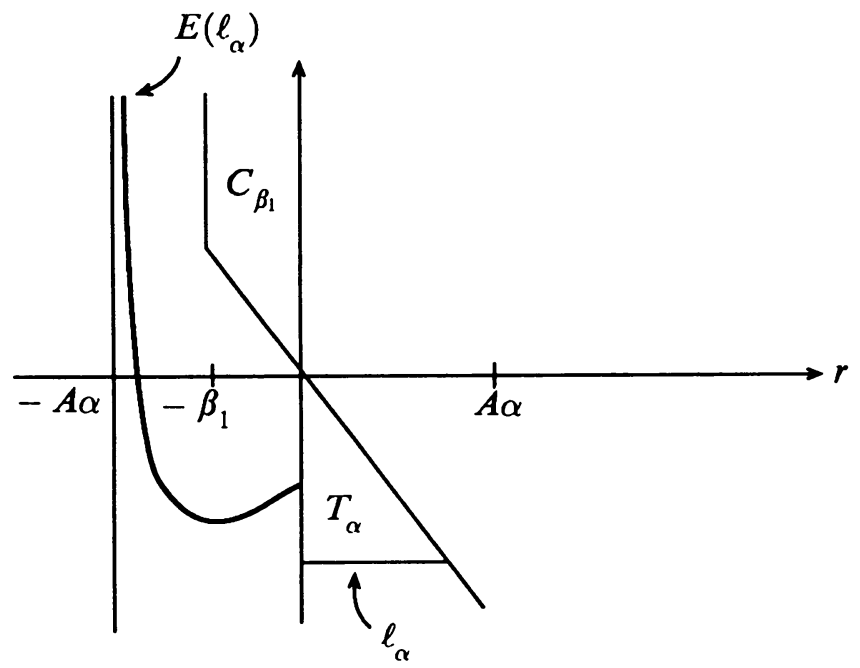

Figure 3. $E\left(\mathscr{L}_{\alpha}\right)$ AND the SUbTRIANGle $T_{\alpha}$.

sufficiently large, say $\alpha=\alpha_{4}$. Finally, let $T_{\alpha} \subset T$ be the subtriangle shown in Figure 3. The above observations show that if $\alpha_{1} \geq \max \left\{\alpha_{3}, \alpha_{4}\right\}$ then $E\left(T_{\alpha}\right) \supset C_{\beta_{1}}$ for $\alpha \geq \alpha_{1}$.

Fix $\alpha=\alpha_{1}$ for some such choice of $\alpha_{1}$. 
Proposition 2. The triangle $T_{\alpha_{1}}$ has a bounded pre-image $T_{\alpha_{1}}^{-1}$ in the right halfplane.

Proof. In Proposition 1 we established the existence of a pre-image in the right half-plane. We need to show that $T_{\alpha_{1}}^{-1}$ is bounded. In Lemma 2 we established an upper bound on the $p$-coordinate of points in $T_{\alpha_{1}}^{-1}$, i.e., $(r, p) \in T_{\alpha_{1}}^{-1}$ implies $p \leq K$ for some $K>0$.

Let $R_{\alpha_{1}}$ denote the rectangle

$$
R_{\alpha_{1}}:\left\{(r, p) \mid 0 \leq r \leq A \alpha_{1},-\alpha_{1} \leq p \leq 0\right\},
$$

where $\alpha_{1}$ is as above. Notice that $R_{\alpha_{1}} \supset T_{\alpha_{1}}$.

Lemma 3. There exists $K_{2}>0$ such that if $p \leq-K_{2}$ then $E(r, p) \notin R_{\alpha_{1}}$.

Proof of Lemma 3. Consider the image of the horizontal line $p=-K_{2}$.

$$
E\left(r,-K_{2}\right)=\left(r-A K_{2},-K_{2}+r^{-3}(B-C r)\right)=\left(r_{2}, p_{2}\right) .
$$

We seek $K_{2}$ such that

$$
p_{2}=-K_{2}+r^{-3}(B-C r)<-\alpha_{1},
$$

that is,

$$
K_{2}>\alpha_{1}+r^{-3}(B-C r) .
$$

In the proof of Lemma 2 we showed that $f_{2}(r)=r^{-3}(B-C r)$ has a global maximum at $r_{*}=3 B / 2 C$. Since $\alpha_{1}$ is a positive constant, we can clearly choose a suitable $K_{2}>0$.

Thus $T_{\alpha_{1}}^{-1}$ must lie in the infinite strip $\left\{r>0,-K_{2} \leq p \leq K\right\}$ where $K$ is as in Lemma 2.

Lemma 4. There exists $K_{1}>0$ such that if $r \geq K_{1}$ and $-K_{2} \leq p \leq K$, then $E(r, p) \notin R_{\alpha_{1}}$.

Proof of Lemma 4. Consider the image of the vertical line segment $r=K_{1}$, $-K_{2} \leq p \leq K$.

$$
E\left(K_{1}, p\right)=\left(K_{1}+A p, p+K_{1}^{-3}\left(B-C K_{1}\right)\right)=\left(r_{1}, p_{1}\right) .
$$

We seek $K_{1}>0$ such that

$$
r_{1}=K_{1}+A p>A \alpha_{1}
$$

that is,

$$
K_{1}>A\left(\alpha_{1}-p\right) .
$$

Since $-K_{2} \leq p \leq K$, we see that $A\left(\alpha_{1}-p\right) \leq A\left(\alpha_{1}+K_{2}\right)$. Thus, for $K_{1}>$ $A\left(\alpha_{1}+K_{2}\right)$, we obtain the conclusion of the lemma.

Lemmas 2, 3, and 4 together imply that $T_{\alpha_{1}}^{-1}$ must lie in the rectangle bounded by $r=0, r=K_{1}, p=-K_{2}$, and $p=K$. Hence $T_{\alpha_{1}}$ has bounded 
pre-image $T_{\alpha_{1}}^{-1}$ in the right half-plane. This concludes the proof of Proposition 2.

Having established that $T_{\alpha_{1}}$ has bounded pre-image, and recalling that $E\left(C_{\beta_{1}}\right)$ covers both $T_{\alpha_{1}}$ and $T_{\alpha_{1}}^{-1}$ (Figure 2), we can select a subchisel,

$$
C_{\beta_{1}, \beta_{2}}:\left\{-\beta_{1} \leq r \leq-\beta_{2}<0\right\} \cap\{(r, p) \mid r<0, p \geq-r / A\},
$$

such that $E\left(C_{\beta_{1}, \beta_{2}}\right) \supset T_{\alpha_{1}} \cup T_{\alpha_{1}}^{-1}$. Since $T_{\alpha_{1}} \cup T_{\alpha_{1}}^{-1}$ are contained in some compact rectangle in the right half-plane, we can select a compact subset $\mathscr{K}_{3}$ of $C_{\beta_{1}, \beta_{2}}$ whose image $E\left(\mathscr{K}_{3}\right)$ still covers $T_{\alpha_{1}} \cup T_{\alpha_{1}}^{-1}$ (Figure 4).

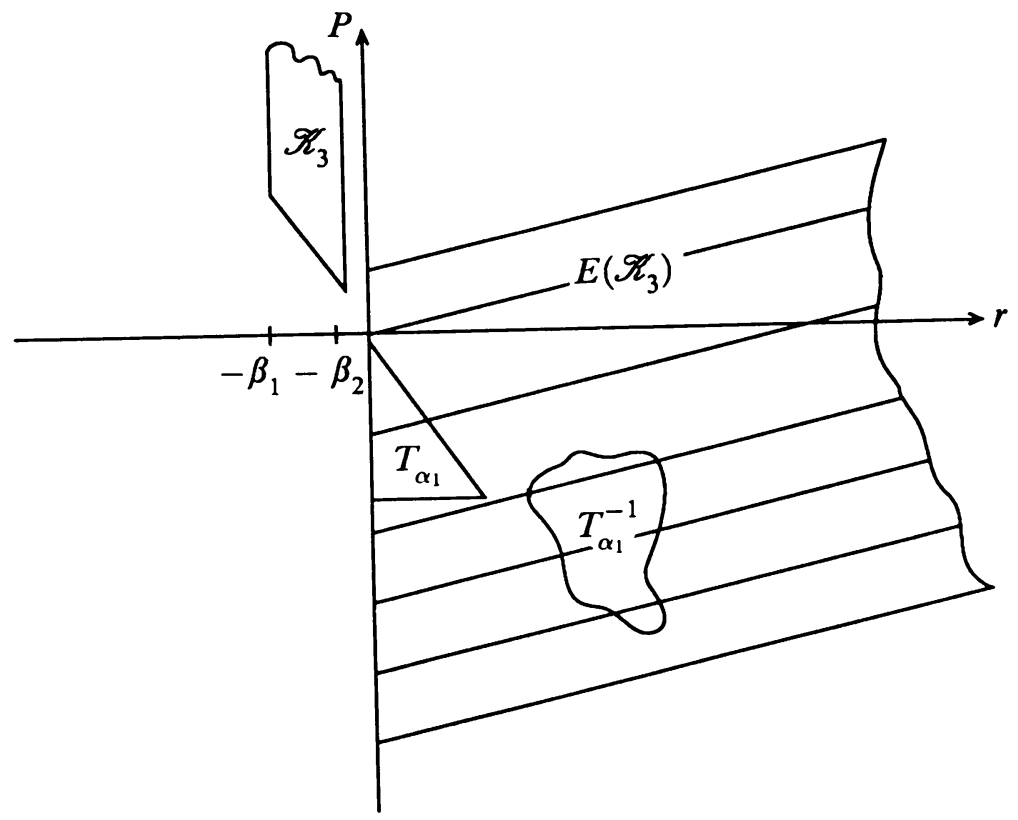

FIGURE 4. THE SET $\mathscr{K}_{3}$.

Next observe that since $E\left(T_{\alpha_{1}}\right) \supset C_{\beta_{1}} \supset \mathscr{K}_{3}$ and $\mathscr{K}_{3}$ is compact, we can select a compact subset $\mathscr{K}_{2} \subset T_{\alpha_{1}}$ whose image $E\left(\mathscr{K}_{2}\right)$ contains $\mathscr{K}_{3}$. Finally, $\mathscr{K}_{2}$ compact implies that its pre-image $\mathscr{K}_{1} \subset T_{\alpha_{1}}^{-1}$ is also compact. Thus we have constructed the desired compact sets $\mathscr{K}_{1}, \mathscr{K}_{2}, \mathscr{K}_{3}$ such that $E\left(\mathscr{K}_{1}\right) \supset$ $\mathscr{K}_{2}, E\left(\mathscr{K}_{2}\right) \supset \mathscr{K}_{3}$, and $E\left(\mathscr{K}_{3}\right) \supset \mathscr{K}_{1} \cup \mathscr{K}_{2}$. This completes the proof of Theorem A. 


\section{Discussion}

The classical approach to numerical simulation of finite dimensional dynamical systems consists of replacing the original system of ordinary differential equations by a discrete mapping which one iterates. One hopes that if the chosen numerical method has a sufficiently small truncation error then, all other things being equal, one need only choose the stepsize small to guarantee accurate results. We have constructed an example which suggests the possibility that, in general, there may be global dynamical phenomona associated with the numerical scheme that cannot be eliminated in this fashion. The results of Lorenz [1988] suggest that the global dynamical behavior of a numerical method is very scheme dependent. Lorenz studies bifurcations which occur as one increases the stepsize of a numerical method as applied to a dynamical system possessing an attractor. These bifurcations are observed to be very method dependent, even for numerical schemes having the same order truncation error.

There is some reason to believe that it will be difficult to obtain general global results such as we have obtained for our example. The main obstruction concerns the behavior of numerical methods under change of coordinates. That is, given a dynamical system

$$
\dot{X}=F(X)
$$

and a smooth change of coordinates

$$
Y=G(X)
$$

which transforms $(4.1)$ into

$$
\dot{Y}=[D G(Y)]^{-1} F(G(Y)),
$$

then the vector fields of (4.1) and (4.3) are equivalent. However, if we apply the same numerical method $\mathscr{N}$ to (4.1) and (4.3), then it is not true in general that the maps $\mathscr{N}(X)$ and $\mathscr{N}(Y)$ are topologically conjugate.

\section{REFERENCES}

1. V. I. Arnold [1978], Mathematical methods of classical mechanics, Springer-Verlag, New York.

2. R. Bowen [1978], On axiom A diffeomorphisms, CBMS Reg. Conf. Ser. in Math.. No. 35, Amer. Math. Soc., Providence, Rhode Island.

3. P.J. Channell and J.C. Scovel [1988], Symplectic integration of Hamiltonian systems, LANL, preprint.

4. J. Curry, L. Garnett and D. Sullivan [1983], On the iteration of a rational function: computer experiments with Newton's method, Comm. Math. Phys., 91, 267-277.

5. F.R. Gantmacher [1960], The theory of matrices, Vol. II, Chelsea Publishing Co., New York.

6. S. Hammel, J. Yorke and C. Grebogi [1988], Numerical orbits of chaotic processes represent true orbits, Bull. Amer. Math. Soc. (New Series), 19, 465-469.

7. E. Lorenz [1988], Computational chaos, MIT, preprint.

8. F. Neri [1988], Lie algebras and canonical integration, University of Maryland, preprint.

9. R. Ruth [1983], A canonical integration technique, IEEE Trans. Nucl. Sci., NS30, 2669. 
10. D. Saari and J. Urenko [1984], Newton's method, circle maps and chaotic motion, Amer. Math. Monthly, 91, 3-17.

11. M. Shub [1987], Global stability of dynamical systems, Springer-Verlag, New York.

12. M. Shub and A. Vasquez [1987], Some linearly induced Morse-Smale systems, the QR algorithm and the Toda lattice, in Contemporary Mathematics, 64, The Legacy of Sonya Kovalevskaya, (Linda Keen, ed.), Amer. Math. Soc., Providence, Rhode Island.

13. S. Smale [1985], On the efficiency of algorithms of analysis, Bull. Amer. Math. Soc. (New Series), 13, 87-121.

Department of Mathematics, The George Washington University, Washington, District of Columbia 20052 